\title{
Optimization Model and Method of Urban Railway Transit Vehicle Use Plan with Long and Short Routing
}

\author{
Dianyuan Huang ${ }^{1,}$ a , Yixiang Yue ${ }^{1, b}$ and Leishan Zhou ${ }^{1, c}$ \\ ${ }^{1}$ School of Traffic and Transportation, Beijing Jiaotong University, Beijing 100044, China \\ ahdy5895@163.com, byueyixiang@163.com, zhouleishan@163.com
}

\begin{abstract}
Keywords: Unban Railway Transit, Improved Assignment Model, Long and Short Routing
Abstract. As one of the most basic transport plan, urban railway transit vehicle use plan has important influence on operating costs and transportation service quality. It is urgent demand to find practical and effective mathematical models and solving method. Based on the development reality of urban railway transit and the advanced experience, this paper make research on optimization model of urban railway transit vehicle use plan and propose rolling time and space assignment model. An example of Shenzhen Metro Line 8 was presented to show the feasibility of the proposed model and algorithm
\end{abstract}

\section{Introduction}

In urban railway transportation process, vehicle use plan is one of the most basic transport plan. In domestic, drawing urban railway transit diagram and vehicle use plan is basically stay half artificial stage, which needs experienced people spend more time and energy[1]. Especially when train dispatching plan change frequently, the number of compile vehicle use plan needs to increase. Therefore, as the main undertaker of public transport passenger flow, on the basis of guarantee the quality of transportation services, how to maximize capacity and improve the utilization efficiency of vehicles is an urgent problem[2].

The form and characteristics of long and short routing. Train routing plan is a train operation plan which needs to consider the requirement of organization and the change of operating conditions. According to different train routings, train routing plan provides for driving section, turn-back station, and train quantity [3]. Therefore, adopting the reasonable and feasible train routing can improve the efficiency of train vehicles use, improve the transport ability, make full use of the urban railway transit system resources, reduce the transportation cost, and can ensure the quality of passenger service.

Nested routing which is also known as long and short routing, means there are two cases of long routing and short routing in the operation. Long routing turn back at the terminal and short routing turn back at the specified middle station. According to the long and short routing overlapping position, two forms can be divided [4]. As the Fig.1 shows.

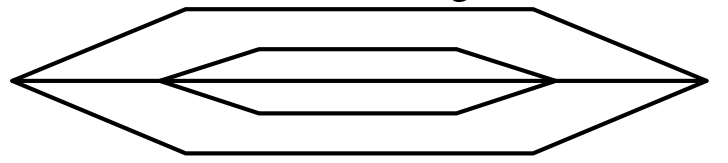

(a)

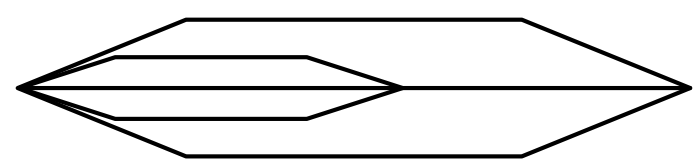

(b)

Fig. 1 The map of the complicated train routing

Long and short routing traffic organization is a kind of economic and reasonable operation scheme, especially with the high degree of segment traffic imbalance, causing a section capacity can't meet the needs of the traffic volume, long and short routing operation organization is particularly applicable. When passenger flow on the spatial distribution is uniform during the peak, and the spatial distribute differently during the through, can also use the train operation plan of long and short routing, organize short routing which part of trains turn back at middle station. However, this kind of traffic organization is relatively complex, needs higher requirements for passenger transport organization level.

Vehicle turnover mode analysis of long and short routing. By the long and short routing form, there are three turnover ways: independently use mode, nested use mode, semi-fixed use mode [5].As is shown in Fig.2 and Fig.3. 


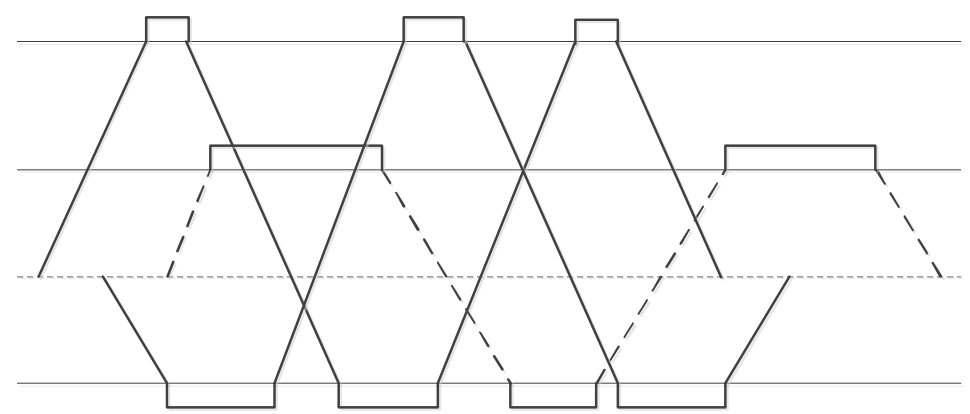

Fig.2 Independently use of vehicles with long and short routing

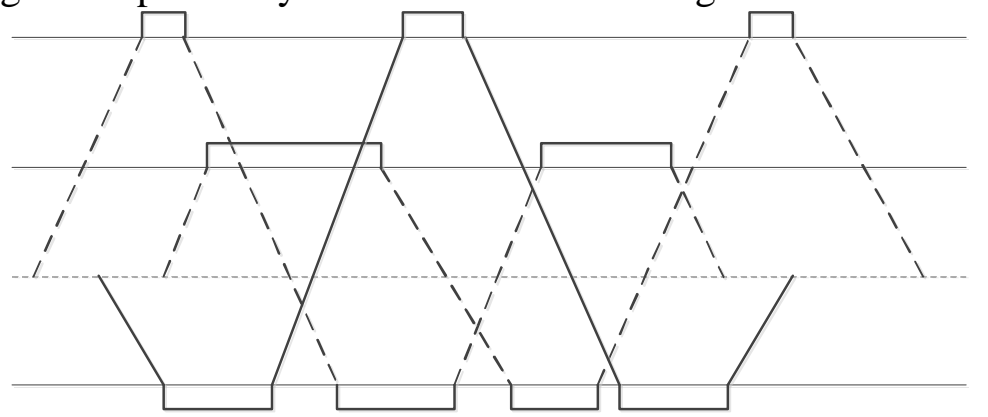

Fig. 3 Nested use of vehicles with long and short routing

Compared with the ordinary routing, long and short routing has the following characteristics:

Compared with the ordinary routing, complicated train routing needs to set up middle turn-back station. The turn-back operation of short routing trains is likely to produce interference with arrival and departure operation of long routing trains.

Use long and short routing operation can improve train loading rate, speed up the train flow, reduce the use of train quantity, thus improve the vehicles use efficiency and reduce operating costs.

Waiting time of part long routing trains' passengers need to be increased. Because, under the condition of invariable total train quantity, short routing trains will occupy block. This increase the long routing trains' departure interval and the long-distance passengers' travel time.

For cross routing passengers, complicated train routing makes them have to transfer to get to the destination, increase transfer organization pressure.

According to the characteristics of the above analysis, easy to get into the applicable scope of the long and short routing nested model. The determination of train routing should be based on the spatial distribution characteristics of passenger flow. In general, long and short routing nested model is suitable for the situation of the passenger flow distribute inequality, and passenger volume is large in the long and short routing overlapping section. At the same time, the proportion of cross passengers and long distance passengers is low [6,7].

\section{Optimization model of vehicle use plan with long and short routing}

Description of the problem. Suppose for urban rail transit line net have $p$ stations, and these stations can accomplish arrival and departure and turn-back operation. Constitute originating and destination stations collection is $N=\left\{N_{1}, N_{2}, \mathrm{~L}, N_{p}\right\}$. There are $n$ train lines on diagram, constitute train lines collection is $L=\left\{L_{1}, L_{2}, \mathrm{~L}, L_{n}\right\}$. Each line has attributes include: starting station name $N_{l}^{f}$, terminal station name $N_{l}^{d}$ block driving time $t_{l}^{y}$, departure time $t_{l}^{f}$, arrival time $t_{l}^{d}$. Vehicle quantity is $m$, constitute vehicle collection is $X=\left\{X_{1}, X_{2}, \mathrm{~L}, X_{m}\right\}$.For vehicle $k$, it's start departure is $t_{k}^{f}$, arrival time is $t_{k}^{d}$, after it finish the task of train line $i$, continue to finish the task of train line $j$, the connecting time at station is $t_{i j}^{k}$.

In constructing the mathematical model of this problem, according to the characteristics of each train line, divide into four collections. Arrival train number collections $\left\{L_{1}^{d}, L_{2}^{d}, \mathrm{~L}, L_{m}^{d}\right\}$, departure depot train 
number collection $\left\{L_{1}^{c d}, L_{2}^{c d}, \mathrm{~L}, L_{n}^{c d}\right\}$, departure train number collections $\left\{L_{1}^{f}, L_{2}^{f}, \mathrm{~L}, L_{p}^{f}\right\}$ and arrival depot train number collection $\left\{L_{1}^{r d}, L_{2}^{r d}, \mathrm{~L}, L_{q}^{r d}\right\}$. Wherein, the arrival train quantity of this period is $m$, the leave depot train quantity of this period is $n$, the leave train quantity of this period is $p$, and the arrival depot train quantity of this period is $q$. On the premise of meet the minimum turn-back time constraints, connect train lines, form a train routing. Then deploy vehicle to complete all the tasks of train lines, and meet the constraints, get the result of vehicle driving mode and using quantity.

The constraint. Constraints of urban rail transit vehicle use questions mainly include three aspects: connecting condition, maintenance condition and turn-back station line ability. This paper considers the urban rail traffic daily operation status, thus weakening maintenance conditions. By add arrival and departure depot vehicle dynamically, can eliminate the need for the turn-back station line ability constraint.

After finish a train line task, a vehicle can only assume the task of a subsequent line. When there is a train line can be connected, vehicle paint a subsequent departure train line. When there is no train line can be connected to this vehicle, make a vehicle arrival depot line. At the same time, delimit this train line into arrival depot train number collection.

$$
\sum_{j=1}^{p} x_{i j}^{f}+\sum_{j=1}^{q} x_{i j}^{r d}=1
$$

Before finish a task of train line, the vehicle can only assume the task of a previous line. When there is a train line can be connected, the arrival of the vehicle connect to a previous train line. When there is no line can be connected to the vehicle, make a vehicle departure depot line. At the same time, delimit this train line into departure depot train number collection.

$$
\sum_{i=1}^{m} x_{i j}^{d}+\sum_{i=1}^{n} x_{i j}^{c d}=1
$$

When an arbitrary train line needs to be connected, meet the requirement of connecting time. $t_{i j}$ is the connecting time of train line $i$ and train line $j, t_{z}$ is the minimum connecting time, $\Delta t$ is the floating up and down time of connecting.

$$
t_{i j} \geq t_{z}-\Delta t
$$

Corresponding relations between vehicle and train line. At the same station, departure time $t_{j}^{f}$ of departure train line $l_{j}$, arrival time $t_{i}^{d}$ of arrival train line $l_{\mathrm{i}}$, arrive depot vehicle's arrive time is $t_{i}^{r d}$, departure depot vehicle's departure time is $t_{j}^{c d}$ :

$$
t_{i j}^{k}= \begin{cases}t_{j}^{f}-t_{i}^{d} & t_{j}^{f}-t_{i}^{d} \geq t_{z} \\ t_{j}^{r d}-t_{i}^{d} & t_{j}^{r d}-t_{i}^{d} \geq t_{z} \\ t_{j}^{f}-t_{i}^{c d} & t_{j}^{f}-t_{i}^{c d} \geq t_{z} \\ M & t_{j}^{f}-t_{i}^{d}<t_{z} \\ M & t_{j}^{r d}-t_{i}^{d}<t_{z} \\ M & t_{j}^{f}-t_{i}^{c d}<t_{z}\end{cases}
$$

$x_{i j}=0$ is a $0-1$ variable, when train line $l_{\mathrm{i}}$ is connected to train line $l_{j}, x_{i j}=1$. Otherwise, $x_{i j}=0 . t_{i j}^{k}$ is when vehicle $k$ have completed the $\operatorname{task}\left\langle l_{i}, l_{j}\right\rangle$, the connection time between train line $i$ and $j$. M is an infinite positive.

The objective function. The target of vehicle use problem is use the minimum quantity of vehicles. So can be expressed as the following form:

$$
X=\left(\sum t_{l}^{y}+\sum t_{l}^{\mathrm{t}}+\sum t_{i j}^{k}\right) * v_{l} / S
$$


$X$ is use vehicles quantity, $\sum t_{l}^{y}$ is total driving time of vehicles, $\sum t_{l}^{t}$ is vehicles' total stop time for passengers get on and get off, $S$ is the longest distance for a vehicle once driving, $v_{l}$ is a vehicle's travel speed.

It is easy to know, only $\sum t_{i j}^{k}$ is uncertain. Calculate the minimum of $X$ is equivalent to calculate the minimum of $\sum t_{i j}^{k}$, the minimum of total connecting time. Therefore, minimum of vehicle quantity can be converted into minimize the total vehicle connection time:

$$
\min Z=\sum_{N} \sum_{\left\langle l_{1}, l_{2}\right\rangle} t_{i j}
$$

$N$ is quantity of departure stations, terminal stations or turn-back stations. This express at all turn-back stations, total connecting time among each train line and other train lines is minimum.

Rolling time and space assignment model. Based on detailed analysis of the above constraints and objective function, can construct rolling time and space assignment model as follows:

$$
\begin{aligned}
& \min z=\sum_{i} \sum_{j} t_{i j} x_{i j}^{d}+\sum_{i} \sum_{j} t_{i j} x_{i j}^{c d}+\sum_{i} \sum_{j} t_{i j} x_{i j}^{f}+\sum_{i} \sum_{j} t_{i j} x_{i j}^{r d} \\
& \text { s.t. } \sum_{i=1}^{m} x_{i j}^{d}+\sum_{i=1}^{n} x_{i j}^{c d}=1 \\
& \sum_{j=1}^{p} x_{i j}^{f}+\sum_{j=1}^{q} x_{i j}^{r d}=1 \\
& t_{i j} \geq t_{z}-\Delta t \\
& t_{i j}^{k}= \begin{cases}t_{j}^{f}-t_{i}^{d} & t_{j}^{f}-t_{i}^{d} \geq t_{z} \\
t_{j}^{r d}-t_{i}^{d} & t_{j}^{r d}-t_{i}^{d} \geq t_{z} \\
t_{j}^{f}-t_{i}^{c d} & t_{j}^{f}-t_{i}^{c d} \geq t_{z} \\
M & t_{j}^{f}-t_{i}^{d}<t_{z} \\
M & t_{j}^{r d}-t_{i}^{d}<t_{z} \\
M & t_{j}^{f}-t_{i}^{c d}<t_{z}\end{cases} \\
& x_{i j}= \begin{cases}1 & l_{i} \text { is connected to } l_{j} \\
0 & \text { other }\end{cases}
\end{aligned}
$$

The formula 1 is objective function. The formula 2 ensure that each train line only is connected to a former line, former line can be arrival train line or departure depot train line. The formula 3 ensure that each vehicle only sketch a subsequent train line, subsequent train line can be departure train line or arrival depot train line. The formula 4 restrict that two connecting train lines can meet the need of minimum connecting time. The formula 5 is the calculation method of each two train lines' connecting time. The formula 6 is when $x_{i j}=1$, train line $i$ is connected with train line $j$, otherwise $x_{i j}=0$.

\section{Case Analysis}

Through the ShenZhen metro line 8 as an example, solve the basic vehicle use plan and make a detailed analysis of the results.

The status quo of vehicle use. ShenZhen metro line 8 use low-speed maglev train and complicated train routing. There are 16 stations in this line, form GuoMao to Xiaomeisha is long routing and form Guomao to Shatou is short routing. As is shown in Fig.4. 


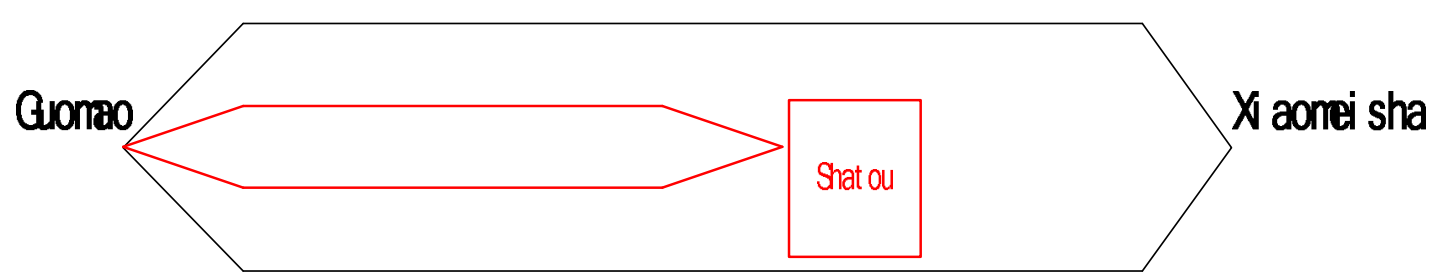

Fig.4 The map of the train routing of Shenzhen Metro Line 8

As can be seen from routing map, length of this line is a little longer than others. This line also pass through Dameisha and Xiaomeisha scenic spots, seasonal characteristics of passenger flow is obvious. Therefore, from the view of saving vehicles, Shenzhen metro line 8 use long and short routing.

In 2016, to the early goal, plan operation 20 pairs of trains. Long routing train and short routing train are 10 pairs. In 2020, medium-term goal, plan operation 24 pairs of trains. Long routing train and short routing train are 12 pairs. In 2030, long-term goal, plan operation 30 pairs of trains, long routing train and short routing train are 15 pairs.

Results analysis. Write a computer program of the algorithm by MATLAB software. This program include 5 functions: Hungary, ZeroNumber, ZeroCover, TryAssign, IsInMatrix. The core function is Hungary function and Hungarian algorithm to solve the assignment problem is mainly reflected in this function. ZeroNumber function is used to calculate the quantity of zero elements of the vector A. ZeroCover function is used to cover all of the zero element with the least amount of straight line. IsInMatrix function is used to determine whether there is elements a in matrix A. TryAssign function try to assign by matrix $\mathrm{C}$ which each line and each column have zero element.Operation process and the results show that the program is a good way to solve the assignment problem. According to the order of time section division, get train connecting plan of each time segment.

For connecting plan matrix, convert to train connecting relationship. Connect result of two neighboring time section, then get train routing plan. According to the above calculation process, get one day's train routing plan. Choose shorter routing, make intersection and combination matching with other short routings, and deploy a vehicle to finish the task. After cross and combinatorial optimization of routing, the quantity of routing reduced from 51 to 24 . At the same time, make train line of each routing is relatively balanced, each vehicle's using rate is higher.

According to the results of vehicle use plan, draw the vehicle use plan picture of Shenzhen metro line 8 by Shenzhen metro line 8 transportation organization management and simulation system. As Fig5.shows.

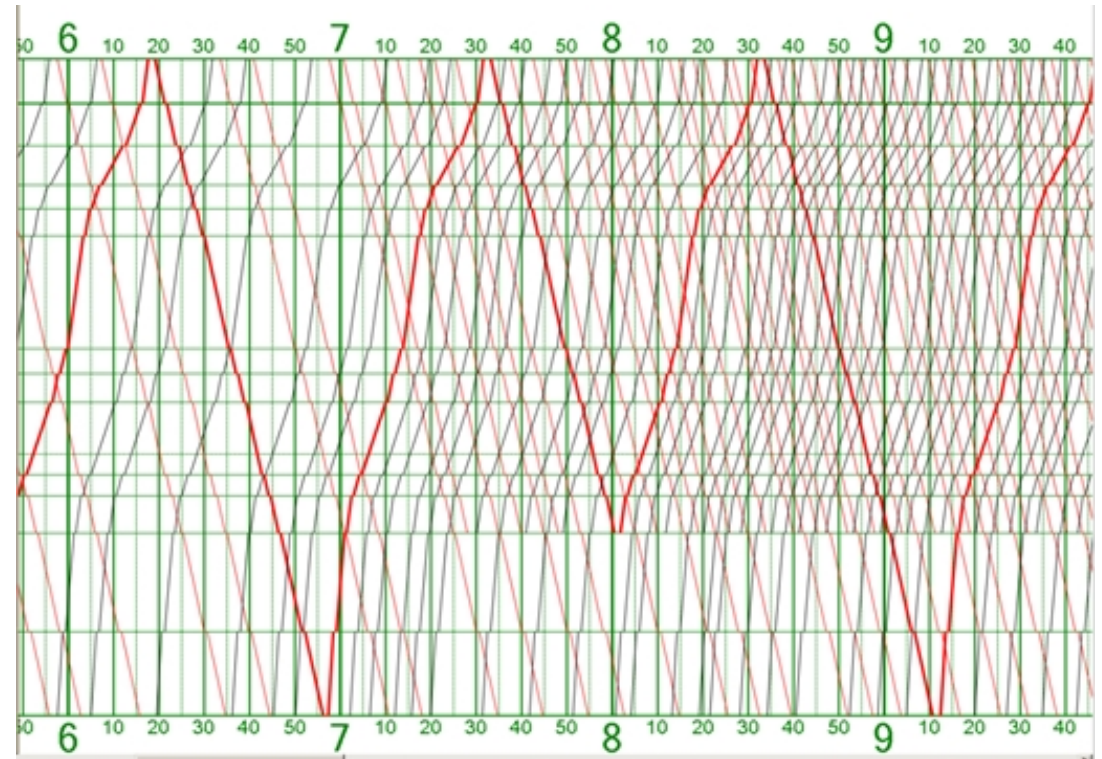

Fig.5 Scheduling of Shenzhen Metro Line 8

In order to measure effect of vehicle use problem by assignment model, make a quantitative analysis by several indicators. 
Quantity of vehicle. Through accumulate times of vehicle arrive or leave depot, can get total using vehicle quantity is 12 pairs.

Turnover time. In this vehicle use example, can calculate all of vehicles' driving time is $880596 \mathrm{~s}$. Average daily turnover time is $36691.5 \mathrm{~s}$, about $11.5 \mathrm{~h}$. Driving time of vehicle is long, save operating costs.

Vehicle daily train mileage. In this vehicle use example, the mileage of short routing is $22.507 \mathrm{~km}$, long routing is $30.630 \mathrm{~km}$. Vehicles can complete 98 short routings and 276 long routings in a day. So can get vehicle daily train mileage is $444.15 \mathrm{~km}$ per vehicle.

Total connecting time. Total connection time is an important indicator that reflect compactness of vehicle use. According to the formula, this example's total connection time is 39364s.

Proportionality of vehicle using. The average using rate is 0.042 , variance of using rate is 0.116 . As can be seen from variance of using rate, each vehicle's using rate is close to the average. It is balanced to adopt this plan.

\section{Conclusions}

Based on the present situation of the urban rail transit vehicle use plan, with long and short routing mode of urban rail transit vehicles use optimization problem as the research object, propose rolling time and space assignment model. However, the practice of urban rail transit transport organization process is complex and job content is various. In addition to daily transport production, may also appear many sorts of emergency. Therefore, to comprehensively and deeply grasp the characteristics of urban rail transit vehicle using, and achieve optimal solution, there are a lot of the follow-up work to do.

\section{References}

[1] Saeed Zolfaghari, Nader Azizi, Mollamad Y. Jaber. A model for holding strategy in Public transit systems with real-time information[J]. International Journal of Transport Management 2,2004:99-110. [2] May, C.The parallel assignment problem redefined[J]. Software Engineering, 1989, 15(6): 821824.

[3] Lefei Li, Han Zhang, Xiaofang Wang, Wei Lu; Zongping Mu. Urban Transit Coordination Using an Artificial Transportation System[J]. Intelligent Transportation Systems, 2011, 12(2): 374- 383.

[4] Chen, J.-F., Lin, R.-L., Liu, Y.-C. Optimization of an MRT Train Schedule: Reducing Maximum Traction Power by Using Genetic Algorithms[J]. Power Systems,2005,20(3):1366-1372.

[5] Khan, M.B.,Xuesong Zhou. Stochastic Optimization Model and Solution Algorithm for Robust Double-Track Train-Timetabling Problem[J]. Intelligent Transportation Systems,2010,11(1):81-89. [6] Shafia, M.A.,Aghaee, M.P., Sadjadi, S.J., Jamili, A. Robust Train Timetabling Problem:

Mathematical Model and Branch and Bound Algorithm[J]. Intelligent Transportation Systems, 2012, 13(1): 307-317.

[7] Zhuan, X., Xia, X. Cruise control scheduling of heavy haul trains[J]. Control Systems Technology, 2006, 14(4): 757-76 